\title{
Plan comparison of volumetric-modulated arc therapy (RapidArc) and conventional intensity- modulated radiation therapy (IMRT) in anal canal cancer
}

Sabine Vieillot ${ }^{1}$, David Azria ${ }^{1 *}$, Claire Lemanski ${ }^{1}$, Carmen Llacer Moscardo ${ }^{1}$, Sophie Gourgou ${ }^{2}$, Jean-Bernard Dubois ${ }^{1}$ , Norbert Aillères ${ }^{1}$, Pascal Fenoglietto ${ }^{1}$

\begin{abstract}
Background: To compare volumetric-modulated arc therapy (RapidArc) plans with conventional intensitymodulated radiation therapy (IMRT) plans in anal canal cancers.

Methods: Ten patients with anal canal carcinoma previously treated with IMRT in our institution were selected for this study. For each patient, three plans were generated with the planning CT scan: one using a fixed beam IMRT, and two plans using the RapidArc technique: a single (RA1) and a double (RA2) modulated arc therapy. The treatment plan was designed to deliver in one process with simultaneous integrated boost (SIB) a dose of 59.4 Gy to the planning target volume (PTV2) based on the gross disease in a 1.8 Gy-daily fraction, 5 days a week. At the same time, the subclinical disease (PTV1) was planned to receive 49.5 Gy in a 1.5 Gy-daily fraction. Plans were normalized to $99 \%$ of the PTV2 that received $95 \%$ of the prescribed dose. Planning objectives were $95 \%$ of the PTV1 will receive $95 \%$ of the prescribed dose and no more than $2 \%$ of the PTV will receive more than $107 \%$. Dosevolume histograms (DVH) for the target volume and the organs at risk (bowel tract, bladder, iliac crests, femoral heads, genitalia/perineum, and healthy tissue) were compared for these different techniques. Monitor units (MU) and delivery treatment time were also reported.
\end{abstract}

Results: All plans achieved fulfilled objectives. Both IMRT and RA2 resulted in superior coverage of PTV than RA1 that was slightly inferior for conformity and homogeneity $(p<0.05)$.

Conformity index ( $\mathrm{Cl}_{95 \%}$ ) for the PTV2 was $1.15 \pm 0.15$ (RA2), $1.28 \pm 0.22$ (IMRT), and $1.79 \pm 0.5$ (RA1). Homogeneity $\left(\mathrm{D}_{5 \%}-\mathrm{D}_{95 \%}\right)$ for PTV2 was $3.21 \pm 1.16$ Gy (RA2), $2.98 \pm 0.7$ Gy (IMRT), and $4.3 \pm 1.3$ Gy (RA1). RapidArc showed to be superior to IMRT in terms of organ at risk sparing. For bowel tract, the mean dose was reduced of 4 Gy by RA2 compared to IMRT. Similar trends were observed for bladder, femoral heads, and genitalia. The DVH of iliac crests and healthy tissue resulted in comparable sparing for the low doses (V10 and V20). Compared to IMRT, mean MUs for each fraction was significantly reduced with RapidArc $(p=0.0002)$ and the treatment time was reduced by a 6-fold extent.

Conclusion: For patients suffering from anal canal cancer, RapidArc with 2 arcs was able to deliver equivalent treatment plan to IMRT in terms of PTV coverage. It provided a better organ at risk sparing and significant reductions of $\mathrm{MU}$ and treatment time per fraction.

\footnotetext{
* Correspondence: azria@valdorel.fnclcc.fr

'Département de Cancérologie Radiothérapie et de Radiophysique, CRLC Val

d'Aurelle-Paul Lamarque, Montpellier, France

Full list of author information is available at the end of the article
} 


\section{Background}

Conventional chemoradiation is the established treatment for anal carcinoma. This organ-preserving approach gives an equivalent cure than radical surgery but at the cost of high acute and late pelvic toxicities. These side-effects can lead to undue treatment breaks and long overall treatment times and therefore may negatively influence outcome [1-3].

Intensity-Modulated Radiotherapy (IMRT) is a treatment delivery technique based on inverse planning optimisation to modulate intensity beams by using multileaf collimator (MLC). During radiation delivery, the leaves are adjusted while the beam is on. IMRT allows the possibility of producing concave dose distributions and providing specific sparing of normal tissue [4]. We performed a dosimetric study about anal canal carcinoma and showed that IMRT resulted in significant reductions in the doses delivered to the bowel, bladder and genitalia/perineal skin [5]. These dosimetric findings were correlated with lower rates of acute GI and GU morbidities and high conformation to the target volume for anal carcinoma [6-10].

We started to treat patients suffering for anal carcinoma with IMRT in May 2007 but we rapidly switch to volumetric modulated arc therapy (VMAT). Indeed VMAT is a new form of IMRT optimisation combining one gantry rotation and the following capabilities: variable dose-rate, variable gantry speed and dynamic MLC [11]. Details of the RapidArc process and quality assurance are detailed in several publications [11,12]. The VMAT approach has a number of potential advantages compared to IMRT: reducing significantly the treatment time and the number of MU, improving normal tissue sparing while keeping the adequate coverage.

In the present study we compared RapidArc with IMRT in anal canal patients including iliac crests sparing measurements.

\section{Methods}

\section{Patient selection, simulation and treatment planning}

Ten patients with localized anal canal carcinoma treated with IMRT in our institution were selected for this study. Five patients were staged II, three IIIA, and two IIIB according to the American Joint Committee on Cancer 2006 Guidelines (AJCC) [13]. Details are shown in Table 1.

For all patients, simulation was performed on computed tomography scan (RT 16 PRO CT Simulator, General Electrics Systems, Cleveland, OH) with a 2.5 $\mathrm{mm}$ thick slices from the mid-dorsal spine to the midfemur. Patients were simulated in the supine position.

The PTV1 included the subclinical and primary disease, with inguinal, perirectal, and pelvic area whereas the PTV2 encompassed the primary disease only. Details of the delineation of these volumes were recently described [5].

The considered organs at risk (OAR) were bowel, bladder, external genitalia/perineal skin (penis and scrotum for men and vulva for women), iliac crests, and femoral heads. For bowel and bladder, a second volume was created and defined as the considered organ minus the PTV (bladder - PTV, bowel - PTV) to avoid hot spots and improve the optimisation. The healthy tissue was defined as the body covered by the CT scan minus the PTV.

The treatment plan was designed to deliver in a single phase process (with simultaneously integrated boost, SIB) a dose of 59.4 Gy to the PTV2 in 33 fractions (1.8Gy daily fractions) and at the same time 49.5 Gy to the PTV1 (1.5-Gy daily fractions).

Considering the radiobiological equivalent dose, 49.5 Gy in 1.5 Gy fractions were considered to be similar to $45 \mathrm{~Gy}$ in $1.8 \mathrm{~Gy}$ fractions using the linear-quadratic model and an $\alpha / \beta=10$.

Once the treatment planning was completed, the plan was normalized to cover $99 \%$ of the PTV2 with $\geq 95 \%$ of the prescribed dose. We also checked that $95 \%$ of the PTV1 received $95 \%$ of the prescribed dose. No more than $2 \%$ of the PTV was allowed to receive more than $107 \%$ of the prescribed dose.

\section{Planning techniques and objectives}

Three sets of plans were generated and compared for this study. All IMRT and RA plans were done using $18 \mathrm{MV}$ photons using a Varian clinac with a 120 leaves Millennium dynamic multileaf collimator (21 EX, Varian, Palo Alto, CA).

\section{IMRT plans}

IMRT plans were generated using commercial inverse planning software (Eclipse, Helios, version 7.2.34, Varian, Palo Alto, CA). Beam geometry consisted of seven coplanar fields for the whole pelvis with the following gantry angles: $0^{\circ}, 45^{\circ}, 110^{\circ}, 180^{\circ}, 250^{\circ}$, and $315^{\circ}$. Default smoothing values were used during optimisation. First optimisation criteria and constraints are detailed in our recent publication [5]. Dose rate (DR) of $300 \mathrm{MU} / \mathrm{min}$ was selected rather than $600 \mathrm{MU} / \mathrm{min}$, in order to decrease mechanical constraints for multileaves collimator (MLC), even if dosimetric results are similar. Calculation was performed with AAA algorithm, and grid of $2.5 \mathrm{~mm}$.

\section{RapidArc plans}

RapidArc optimisation was performed with the version 8.6.05 from Eclipse, (Helios, Varian, Palo Alto, CA). The 
Table 1 Tumor staging, PTV and bowel volumes

\begin{tabular}{ccccccccccc}
\hline Patient & $\mathbf{1}$ & $\mathbf{2}$ & $\mathbf{3}$ & $\mathbf{4}$ & $\mathbf{5}$ & $\mathbf{6}$ & $\mathbf{7}$ & $\mathbf{8}$ & $\mathbf{9}$ & $\mathbf{1 0}$ \\
\hline AJCC stage & $\|$ & $\|$ & $\|$ & $\|$ & $\|$ & IIIA & IIIA & IIIA & IIIB & IIIB \\
TNM stage & T2NO & T2N0 & T2NO & T3NO & T3NO & T2N1 & T4N0 & T3N1 & T2N2 & T3N2 \\
PTV1 volume & 2187 & 2010 & 1933 & 2010 & 2082 & 1775 & 1935 & 1916 & 2770 & 2160 \\
PTV2 volume & 295 & 203 & 141 & 125 & 445 & 293 & 238 & 122 & 279 & 407 \\
Bowel volume & 650 & 587 & 606 & 226 & 385,5 & 149 & 51,5 & 495,5 & 450 & 1011 \\
Bowel-PTV volume & 265 & 219 & 343 & 188 & 268 & 117 & 36 & 302 & 386 & 662 \\
\hline
\end{tabular}

maximum DR of $600 \mathrm{MU} / \mathrm{min}$ was selected. Starting optimisation constraints consisted in the results of IMRT plans. RapidArc with 1 arc (RA1) corresponded to a single $360^{\circ}$ rotation and RapidArc with 2 arcs (RA2) to two coplanars arcs of $360^{\circ}$ sharing the same isocenter and optimised independently and simultaneously. These two arcs were delivered with opposite rotation (clock and counter-clock) and so minimize the off-treatment between the two beams time about 25 seconds.

For RA1, field size and collimator rotation were determined by the automatic tool from Eclipse to encompass the PTV. We controlled that the collimator was always rotated to a value different from zero in order to avoid tongue and groove effect.

For RA2, the first arc was similar to the one defined in the RA1 process except for the rotation of the collimator, which was 360-X for the second arc (X corresponded to the rotation of the collimator of the first arc).

To improve the results, we tried to modify constraints and priority factors on IMRT and RA plans. These parameters were modified in function of DVH results for each patient.

When necessary, field size was minimized to $15 \mathrm{~cm}$ in the $\mathrm{X}$ direction. This dimension corresponded of the maximal displacement of a leave in a MLC Bank. Doing that, all the leaves positions were possible during the optimisation process increasing the degree of modulation even if in beam eye view a part of the volume was excluded of the beam at each gantry position. Globally rotational delivery permitted to irradiate all the volume of the PTV during rotation of the Linac.

\section{Evaluation tools}

Dose Volume Histograms (DVH) were generated to evaluate the three different plans.

For PTV, the parameters D2\% and D98\% were used as surrogate markers for maximum and minimum doses. Mean dose (D mean) was also reported.

The degree of conformity of the plans was defined as the ratio between the volume receiving at least $95 \%$ of the prescribed dose and the volume of the PTV $\left(\mathrm{CI}_{95 \%}\right)$.
The homogeneity index (HI) was expressed by D5\% D95\% (difference between the dose covering 5\% and $95 \%$ of the PTV).

For all patients DVH for OAR (bowel, bladder, femoral heads, and ilac crests) were calculated and compared. A set of Vx values and D mean was therefore reported.

For healthy tissue, we detailed the volume of the body minus PTV receiving low doses (V5, V10, and V20 Gy).

The number of Monitor Units per fraction required for each plan and the treatment delivery time (from start to the end of the irradiation) was reported.

Treatment techniques were compared using a Mann and Whitney test with significant differences at the $\mathrm{p}<$ 0.05 level.

\section{Results}

PTV volumes, Target coverage, conformity, and dose homogeneity

The mean PTV1 and PTV2 were $2048 \pm 305$ cc (range, 1619-2770) and $255 \pm 112$ cc (range, 122-445), respectively. For the bowel, the mean volume was $461 \pm 279$ cc (range: 51.6-1011.4). Table 1 shows the different volumes (PTV, bowel, and bowel-PTV) delineated for the 10 patients and the results for PTV in terms of coverage and conformity are listed in Table 2.

All the planning objectives were achieved with the three plans. RA1 reached higher values for the maximum significant dose (D2\%) compared to IMRT ( $\mathrm{p}=$ $0.004)$ or RA2 $(\mathrm{p}=0.01)$.

RA2 and IMRT were equivalent for conformity and homogeneity index with a non significant trend for better results with RA2 $(\mathrm{p}=0.09)$. RA1 showed to be slightly inferior to both IMRT and RA2 for these indices $(\mathrm{p}<0.05)$. Figure 1 depicts dose distribution for IMRT and RA2.

\section{Organs at risk}

Table 3 details numerical findings.

The bowel DVH parameters (V49.5 V45, V40, V30) were similar for the three plans. The mean dose was reduced by 4.7 Gy with RA2 compared to IMRT, (IMRT: $38.8 \pm 12.9$ vs $34.1 \pm 17.7$ Gy, p = NS). 
Table 2 Dosimetric results for PTV1 (49.5 Gy) and PTV2 (59.4 Gy)

\begin{tabular}{|c|c|c|c|c|}
\hline & PLANS & IMRT & RA1 & RA2 \\
\hline \multirow[t]{6}{*}{ PTV1 } & D98\%, Gy (\%) & $47.1 \pm 0.8(95.2)$ & $46.6 \pm 1.2(94.2)$ & $46.3 \pm 0.9(93.7)$ \\
\hline & D95\%, Gy (\%) & $47.7 \pm 0.8(96.4)$ & $47.8 \pm 1.2(96.6)$ & $47.4 \pm 0.8(95.8)$ \\
\hline & D2\%, Gy (\%) & $59.8 \pm 0.7(120)$ & $61.6 \pm 1.6(124)^{a, b}$ & $60.1 \pm 1.3(121)$ \\
\hline & HI, Gy (D5\%-D95\%) & $11.2 \pm 0.6$ & $12.8 \pm 1.1^{\mathrm{a}}$ & $15 \pm 1.4$ \\
\hline & $\mathrm{Cl}$ & $1.2 \pm 0.1$ & $1.2 \pm 0.1$ & $1.17 \pm 0.1$ \\
\hline & Dmean, Gy (\%) & $52.1 \pm 1.5(105)$ & $53.32 \pm 1.7(107)$ & $52.2 \pm 1(105)$ \\
\hline \multirow[t]{6}{*}{ PTV2 } & D98\%, Gy (\%) & $56.8 \pm 0.13(95.6)$ & $57 \pm 0.2(95.9)^{a, b}$ & $56.8 \pm 0.1(95.6)$ \\
\hline & D95\%, Gy (\%) & $57.4 \pm 0.3(96.5)$ & $57.8 \pm 0.5(97.4)^{a, b}$ & $57.4 \pm 0.3(96.5)$ \\
\hline & D2\%, Gy (\%) & $60.6 \pm 0.98(102)$ & $62.7 \pm 1.64(105)^{\mathrm{a}, \mathrm{b}}$ & $60.8 \pm 1.3(102)$ \\
\hline & HI, Gy (D5\%-D95\%) & $3.0 \pm 0.7$ & $4.3 \pm 1.3^{\mathrm{a}, \mathrm{b}}$ & $3.21 \pm 1.16$ \\
\hline & $\mathrm{Cl}$ & $1.2 \pm 0.21$ & $1.8 \pm 0.5^{\mathrm{a}, \mathrm{b}}$ & $1.15 \pm 0.15$ \\
\hline & Dmean, Gy (\%) & $58.9 \pm 0.4(99.2)$ & $60.3 \pm 1.1(101.6)^{a}$ & $59.2 \pm 0.9(99.7)$ \\
\hline
\end{tabular}

$a$ if the difference with IMRT is significant.

$b$ if the difference with RA2 is significant for RA1.

PTV: Planning Target Volume, Dx\%: Dose received by $\mathrm{x} \%$ of the volume, HI: Homogeneity Index, Cl: Conformity Index, D mean: Dose mean.

The plans for the bladder were also equivalent. The volume of bladder irradiated at low and medium doses was reduced with RA2 compared to IMRT with a trend towards statistical significance $(\mathrm{p}=0.07)$.

The same trends were observed for skin and genitalia with a decrease of the irradiated volume to the low and medium doses (V30: IMRT: $21.7 \pm 22.4$ Gy vs RA2: $9 \pm$ $15.1 \mathrm{~Gy}, \mathrm{p}=0.08)$. The mean dose reduced by $5.6 \mathrm{~Gy}$ (IMRT: $24.5 \pm 10.9$ vs $18.9 \pm 6.9$ Gy, $\mathrm{p}=\mathrm{NS}$ )
Considering the (i) femoral heads and (ii) the iliac crest,(i) the mean dose was reduced by 3 Gy with a significant statistical difference $(\mathrm{p}=0.03)$. V45 was inferior to $5 \%$ for all techniques. (ii) No significant difference between the plans was noted for the parameters V10, V20, and mean dose.

Finally, the planning objectives for healthy tissue consisted in minimize the dose, in particular the low doses about 5, 10, and 20 Gy. IMRT and RA plans showed similar results.
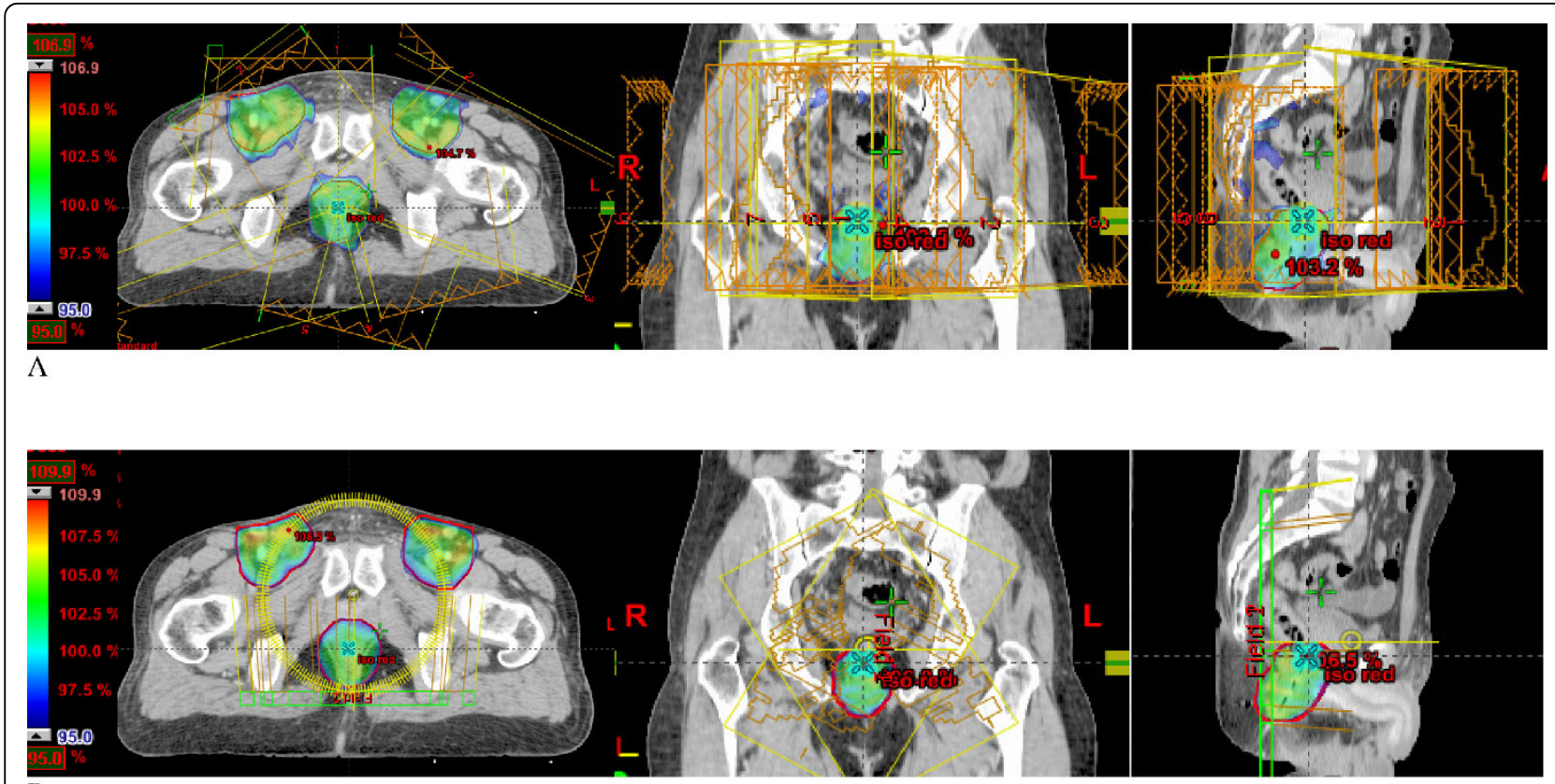
B

Figure 1 Dose distribution by A: Intensity Modulated Radiation Therapy (IMRT) and B: Volumetric Modulated Arc Therapy (VMAT) RapidArc*. 
Table 3 Dosimetric results for organs at risk

\begin{tabular}{|c|c|c|c|c|}
\hline organs & plans & IMRT & RA1 & RA2 \\
\hline \multirow[t]{5}{*}{ bowel } & Dmean (Gy) & $30.4 \pm 7.8$ & $29.9 \pm 8.4$ & $27.8 \pm 7.3$ \\
\hline & V30\% (cc) & $70 \pm 26.5(343 \pm 217)$ & $70 \pm 27(343 \pm 268)$ & $61.8 \pm 24.8(314 \pm 255)$ \\
\hline & V40\% (cc) & $47.7 \pm 29.5(248 \pm 182)$ & $49.5 \pm 23(252 \pm 203)$ & $42.7 \pm 20.8(224 \pm 118)$ \\
\hline & V45\% (cc) & $35.3 \pm 21(193 \pm 155)$ & $36.4 \pm 19.8(192 \pm 159)$ & $31.5 \pm 17.7(171 \pm 150)$ \\
\hline & $\mathrm{V} 49.5 \%(\mathrm{cc})$ & $9.8 \pm 15.8(63 \pm 95)$ & $14.9 \pm 15.6(84 \pm 99)$ & $10.4 \pm 11(58 \pm 82$ \\
\hline \multirow[t]{4}{*}{ bladder } & Dmean (Gy) & $39.1 \pm 4.2$ & $38.8 \pm 4.2$ & $37.5 \pm 5.4$ \\
\hline & V30\% & $81.9 \pm 15.1$ & $80.4 \pm 13.6$ & $70.3 \pm 19.4$ \\
\hline & V40\% & $46 \pm 17.3$ & $45.6 \pm 17.2$ & $40.2 \pm 17.2$ \\
\hline & V45\% & $30.8 \pm 17$ & $30.1 \pm 16.1$ & $26.9 \pm 15.5$ \\
\hline \multirow[t]{4}{*}{ genitalia } & Dmean (Gy) & $24.5 \pm 10.9$ & $19.4 \pm 7.3$ & $18.9 \pm 6.9$ \\
\hline & V20\% & $62.1 \pm 33$ & $51.3 \pm 31.6$ & $48 \pm 31$ \\
\hline & V30\% & $21.7 \pm 22.4$ & $11.8 \pm 17.3$ & $9 \pm 15.1$ \\
\hline & & $2.3 \pm 6,2$ & $1.7 \pm 4.9$ & $1.2 \pm 3.6$ \\
\hline \multirow[t]{3}{*}{ Iliac crests } & Dmean (Gy) & $20.4 \pm 7.2$ & $20.6 \pm 5.2$ & $19.6 \pm 4.4$ \\
\hline & V10\% & $55.3 \pm 15.7$ & $60.1 \pm 15.9$ & $58.9 \pm 18.2$ \\
\hline & V20\% & $37.6 \pm 9,2$ & $45.25 \pm 13.9$ & $40.6 \pm 10.5$ \\
\hline \multirow[t]{4}{*}{ Femoral heads } & D mean (Gy) & $27.5 \pm 2.4$ & $26.2 \pm 2.2$ & $24.6 \pm 4.1$ \\
\hline & V $45 \%$ & $2.2 \pm 3,3$ & $2.2 \pm 2.3$ & $1.7 \pm 1.9$ \\
\hline & Dmean (Gy) & $27.2 \pm 2.4$ & $26.85 \pm 2.9$ & $24.1 \pm 3.2^{\mathrm{a}}$ \\
\hline & V45\% & $0.8 \pm 1$ & $1.7 \pm 1.9$ & $1.3 \pm 1.5$ \\
\hline \multirow[t]{3}{*}{ Healthy tissue } & V5\% & $55.7 \pm 5.4$ & $57 \pm 5.1$ & $57.6 \pm 5.1$ \\
\hline & V10\% & $47 \pm 5$ & $46.7 \pm 6$ & $47.2 \pm 5.7$ \\
\hline & V20\% & $31.9 \pm 3.9$ & $33.4 \pm 7$ & $28.8 \pm 4.8$ \\
\hline
\end{tabular}

IMRT: Intensity Modulated Radiation Therapy, Vx\%: Volume receiving $\mathrm{x} \%$ of the prescribed dose, D mean: Dose mean a the difference between RA2 and IMRT is significant.

We reported DVH results for one patient in figures 2, 3 and 4.

\section{Monitor Units and Delivery time}

The IMRT plans required a mean of $1646 \pm 332$ MUs per fraction whereas the RA1 plans required $80 \%$ less $(330 \pm$ 52 MUs, $\mathrm{p}=0.0002$ ). The use of two arcs resulted to an slight increase of the number of MU $(493 \pm 66)$ compared to one arc $(\mathrm{p}=0.0003)$. The difference between RA2 and IMRT remained significant $(\mathrm{p}=0.0013)$.

Compared to a delivery in $14 \mathrm{~min}$ for IMRT, treatment time (defined as the start to the end of the irradiation) with RA was definitely shorter and was 1.1 and 2.3 minutes for one and two arcs, respectively.

\section{Discussion}

Recent progresses of new technologies in RT are of great interest for quality of treatment and avoidance of toxicities for miscellaneous localizations, in particular for anal canal cancer.
We initially performed a dosimetric study to compare standard RT3D with IMRT showing a significant decrease of the irradiated volume of the OAR, especially for the bone marrow, while keeping an excellent coverage of the PTV [5]. Based on these results we decided to treat patients suffering from anal canal cancer with IMRT as a standard with promising results in terms of acute toxicities [7-10]. RapidArc is a promising technique, providing a coverage of the target volume and spare of organs at risk at least equivalent to IMRT, while it could reduce significantly the treatment time and the number of MU required [14-22].

In the present study, RapidArc proved to be equivalent to IMRT for targeting coverage of anal canal but showed better organ sparing in terms of mean dose. We also confirmed that RapidArc with two arcs (RA2) achieve better results than 1 arc (RA1) for consequent or complex target volume in terms of conformity and homogeneity $[14,17]$. Two arcs allowed superior modulation factor during optimisation due to the independent 


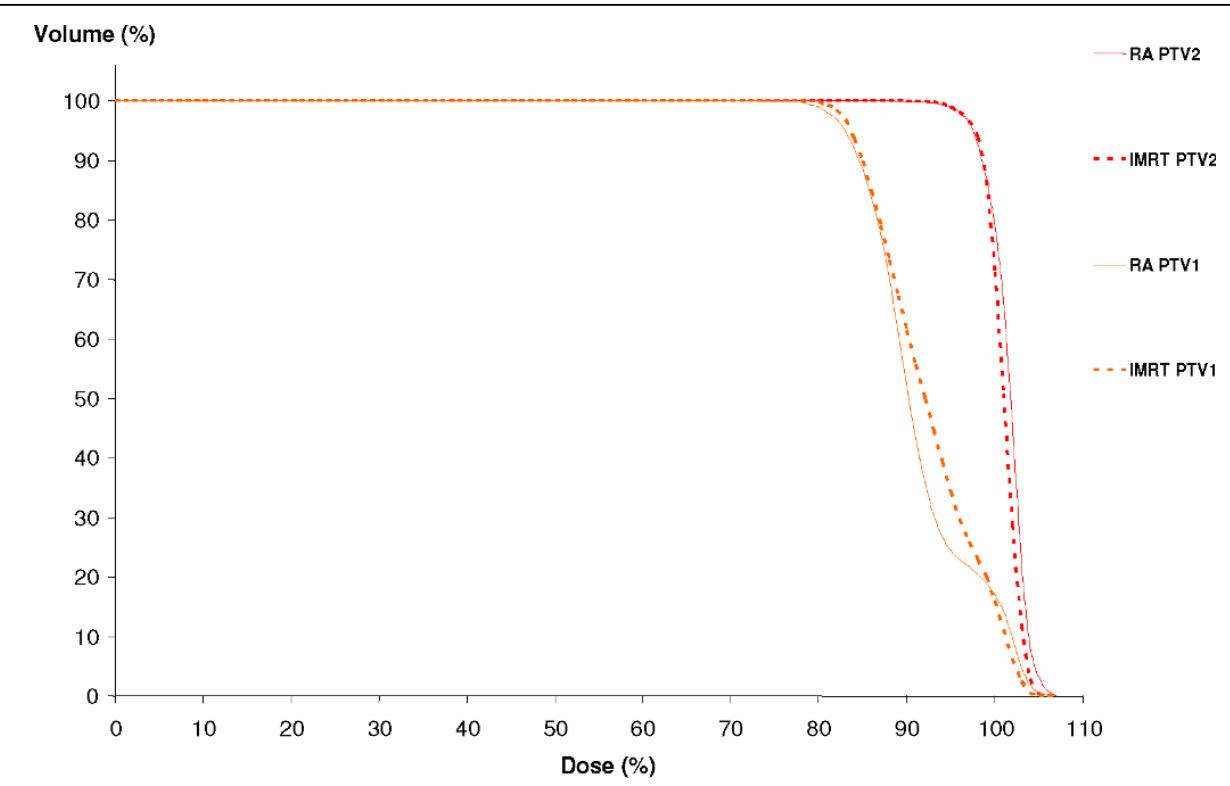

Figure 2 Dose-volume histograms for PTV. RA, Rapidarc; IMRT, intensity-modulated radiotherapy

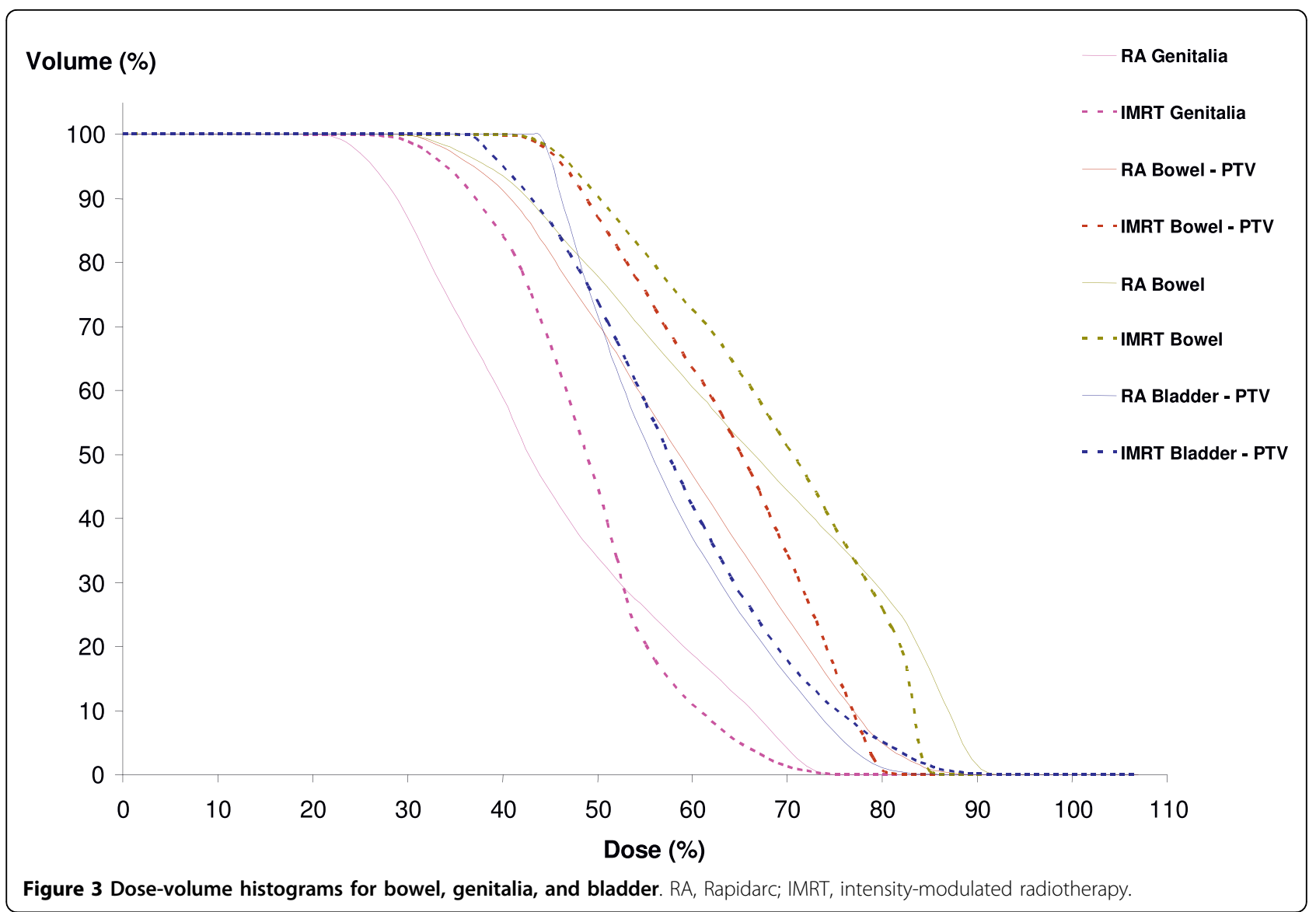




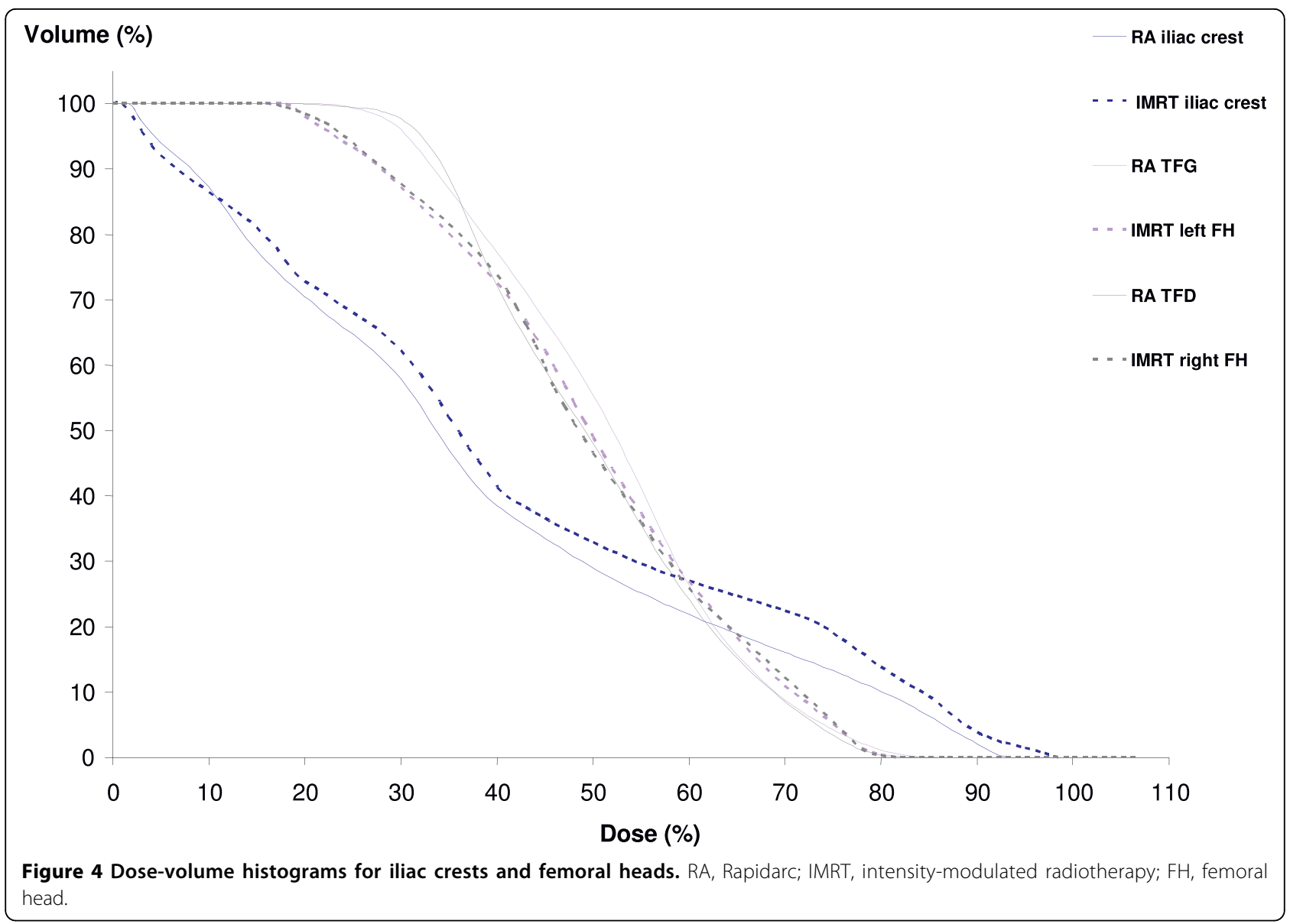

optimisation, and unrelated sequence of MLC shape, gantry speed and dose rate combinations. Some particular options have to be considered for better modulation compared to one arc, particularly the decrease of the MLC in the X direction allowing the optimization process. This approach allows better homogeneity un the target volume and erases the hot spots outside the PTV compared to IMRT (Figure 1).

Clivio et al.[14] published also similar results for PTV but showed different benefit for the OAR, namely for the bowel tract. In one hand the PTV volumes are higher in our series (PTV1 minus PTV2 $=1793 \pm 283$ cc vs $1307 \pm 355 \mathrm{cc}$ ) due to a $1-\mathrm{cm}$ margin from the CTV to the PTV (8 $\mathrm{mm}$ for Clivio et al.). We also delineated the lymph node CTV as recommended by Taylor and al. [23] using a margin of $1 \mathrm{~cm}$ around the vessels. In the other hand, we found lower bowel volumes (461 \pm 279 cc vs. $2483 \pm 774 \mathrm{cc}$ ). Indeed, Gallagher et al. estimated that $1 / 3$ of the small intestine volume would correspond to $660 \mathrm{cc}$ leading to a maximal entire volume of 2000 cc [24].

Our mean irradiated volume above 30 Gy was about $314 \mathrm{cc}$ with RA2 but $844 \mathrm{cc}$ in the Clivio et al. report
[14]. Regarding correlation between dosimetric parameters and acute toxicity, Devisetty et al. [9] showed higher acute GI toxicity for V30 > $450 \mathrm{cc}$ and $\leq 450 \mathrm{cc}$ ( $33 \%$ vs. $8 \%, p=0.003$, respectively).

Regarding acute hematologic toxicity, Mell et al. suggested an association between dosimetric parameters for iliac crests and acute hematologic toxicities, especially in the range of low doses (10 and $20 \mathrm{Gy)}$ [25-27]. We did not find a significant difference between IMRT and RA for these values shown acceptable when compared with 3D [5]. Other dosimetric parameters were also interesting with constraints for bone marrow given for higher doses in the RTOG 0529 protocol (V30, V40, and V50) [28] showing only $23 \%$ grade $3-4$ leucopenia [10].

Considering late toxicities, we referred to the publication of Emami et al. [29] asking for a TD 5/5 (probability of developing $5 \%$ of chronic toxicity within 5 years) for bowel, bladder, and femoral heads. Long-term follow-up are warranted before drawing definitive conclusions.

One of particular interest of RapidArc is the reduction of the time to deliver each fraction and the number of required MU $[14,15,18,30]$. IMRT plans presented in this study were wider than $15 \mathrm{~cm}$ in the direction of the 
MLC motion necessitating splitting into 2 sequences and doubling the number of fields. Dose rate about 600 $\mathrm{MU} / \mathrm{min}$ for IMRT would reduce the beam on time, but not the effective treatment time, which is mainly due to the multiples beams and the "off-time" necessary to move from one to another. The number of MU required is higher due to the sliding window technique. A "step and shoot" technique might lead to lower values. By contrast, treatment with RA is performed simultaneously with rotation by a dynamic MLC adaptation to the target structure during the rotation (the open surface is more important than for sliding window) which reduces the number of MU. For two arcs, the rotation in clock and counter-clock directions allows minimized off-time ( 25 seconds between the 2 arcs). There is no doubt that the reduced treatment time may impact on the treatment quality avoiding long and uncomfortable treatment for the patient and reducing the risk of internal organ motion during the fraction. In addition, more time can be spared for on-line control imaging. A such prolonged fraction delivery time may also have an impact on treatment outcome, due to the increase in cell survival by recovery from sub lethal damage $[31,32]$.

One of the downfalls of IMRT is the potential risk of second cancer [33-36]. Theoretically, the significant reduction of MU with RapidArc decreases scattered dose and may reduce the risk of secondary malignancy. The impact of irradiation of healthy tissue at low doses remains unresolved with the use of RapidArc even if this technique is capable to reduce medium and integral body doses $[15,30]$.

\section{Conclusion}

Compared to IMRT, RapidArc provides an equivalent coverage of PTV and OAR sparing while reducing the number of MU and the treatment time delivery. These improvements have led us to implement rapidly this technique into the clinic. Nine patients have already been treated with RapidArc using two arcs.

\section{List of abbreviations}

3D-CRT: three-dimensional conformal radiation therapy; Cl: Conformity Index; CTV: Clinical Target Volume; D mean: Dose mean; DVH: Dose-volume histograms; DR: Dose rate; HI: Homogeneity Index; IMRT: intensity-modulated radiation therapy; MLC: Multileaf collimator; MU: Monitor units; OAR: Organs at risk; PTV: planning target volume; RA: RapidArc; SIB: simultaneous integrated boost; VMAT: Volumetric Modulated Arc Therapy, Vx\%: Volume receiving $\mathrm{x} \%$ of the prescribed dose

\section{Author details}

'Département de Cancérologie Radiothérapie et de Radiophysique, CRLC Val d'Aurelle-Paul Lamarque, Montpellier, France. ${ }^{2}$ Unité de Biostatistiques, CRLC Val d'Aurelle-Paul Lamarque, Montpellier, France.

\section{Authors' contributions}

SV, PF conceived the study, collected data, and drafted the manuscript. NA $C L M, J B D, C L$, and $D A$ participated in coordination and helped to draft the manuscript. SG performed the statistical analyses. DA provided mentorship and edited the manuscript. All authors have read and approved the final manuscript.

\section{Competing interests}

The authors declare that they have no competing interests.

Received: 2 August 2010 Accepted: 13 October 2010 Published: 13 October 2010

\section{References}

1. UKCCCR Anal Cancer Trial Working Party. UK Co-ordinating Comittee on Cancer Research: Epidermoid anal cancer: results from the UKCCCR randomised trial of radiotherapy alone versus radiotherapy, 5fluorouracil, and mitomycin. Lancet 1996, 348:1049-1054.

2. Bartelink H, Roelofsen F, Eschwege F, Rougier P, Bosset JF, Gonzalez DG, Peiffert D, van Glabbeke M, Pierart M: Concomitant radiotherapy and chemotherapy is superior to radiotherapy alone in the treatment of locally advanced anal cancer: results of a phase III randomized trial of the European Organization for Research and Treatment of Cancer Radiotherapy and Gastrointestinal Cooperative Groups. J Clin Oncol 1997, 15:2040-2049.

3. Flam M, John M, Pajak TF, Petrelli N, Myerson R, Doggett S, Quivey J, Rotman M, Kerman H, Coia L, et al: Role of mitomycin in combination with fluorouracil and radiotherapy, and of salvage chemoradiation in the definitive nonsurgical treatment of epidermoid carcinoma of the anal canal: results of a phase III randomized intergroup study. J Clin Oncol 1996, 14:2527-2539.

4. Intensity modulated radiation therapy collaborative working group: Intensity modulated radiotherapy: current status and issues of interest. Int J Radiat Oncol Biol Phys 2001, 51:880-914.

5. Menkarios C, Azria D, Laliberte B, Moscardo CL, Gourgou S, Lemanski C, Dubois JB, Ailleres N, Fenoglietto P: Optimal organ-sparing intensitymodulated radiation therapy (IMRT) regimen for the treatment of locally advanced anal canal carcinoma: a comparison of conventional and IMRT plans. Radiat Oncol 2007, 2:41.

6. Hodges JC, Das P, Eng C, Reish AG, Beddar AS, Delclos ME, Krishnan S, Crane CH: Intensity-Modulated Radiation Therapy for the Treatment of Squamous Cell Anal Cancer with Para-aortic Nodal Involvement. Int J Radiat Oncol Biol Phys 2009, 75:791-794.

7. Milano MT, Jani AB, Farrey KJ, Rash C, Heimann R, Chmura SJ: Intensitymodulated radiation therapy (IMRT) in the treatment of anal cancer: toxicity and clinical outcome. Int J Radiat Oncol Biol Phys 2005, 63:354-361.

8. Salama JK, Mell LK, Schomas DA, Miller RC, Devisetty K, Jani AB, Mundt AJ, Roeske JC, Liauw SL, Chmura SJ: Concurrent chemotherapy and intensitymodulated radiation therapy for anal canal cancer patients: a multicenter experience. J Clin Oncol 2007, 25:4581-4586.

9. Devisetty K, Mell LK, Salama JK, Schomas DA, Miller RC, Jani AB, Roeske JC, Aydogan B, Chmura SJ: A multi-institutional acute gastrointestinal toxicity analysis of anal cancer patients treated with concurrent intensitymodulated radiation therapy (IMRT) and chemotherapy. Radiother Oncol 2009, 93.298-301.

10. Pepek JMWCG, Clough RW, Wu QJ, Yoo S, Czito BG: Intensity Modulated Radiation Therapy (IMRT) for Anal Cancer: The Duke University Experience. Int J Radiat Oncol Biol Phys 2009, S 2191.

11. Otto K: Volumetric modulated arc therapy: IMRT in a single gantry arc. Med Phys 2008, 35:310-317.

12. Ling CC, Zhang P, Archambault Y, Bocanek J, Tang G, Losasso T: Commissioning and quality assurance of RapidArc radiotherapy delivery system. Int J Radiat Oncol Biol Phys 2008, 72:575-581.

13. Greene $F L$, Page $D L$, Fleming ID, Fritz A, Balch $C M$, Haller DG, Morrow M, (eds): AJCC: Cancer staging manual. New York, 62002.

14. Clivio A, Fogliata A, Franzetti-Pellanda A, Nicolini G, Vanetti E, Wyttenbach R, Cozzi L: Volumetric-modulated arc radiotherapy for carcinomas of the anal canal: A treatment planning comparison with fixed field IMRT. Radiother Oncol 2009, 92:118-24. 
15. Fogliata A, Clivio A, Nicolini G, Vanetti E, Cozzi L: Intensity modulation with photons for benign intracranial tumours: a planning comparison of volumetric single arc, helical arc and fixed gantry techniques. Radiother Oncol 2008, 89:254-262.

16. Palma D, Vollans E, James K, Nakano S, Moiseenko V, Shaffer R, McKenzie M, Morris J, Otto K: Volumetric modulated arc therapy for delivery of prostate radiotherapy: comparison with intensity-modulated radiotherapy and three-dimensional conformal radiotherapy. Int J Radiat Oncol Biol Phys 2008, 72:996-1001.

17. Alexander AS, Wells D, Berrang T, Parsons C, Mydin A, Shaffer R, Wong F, Sayers D, Otto k: Volumetric arc therapy (VMAT) reduces treatment time compared to conventional IMRT (cIMRT) while maintaining similar plan quality in whole pelvic gynecologic radiotherapy. Int J Radiat Oncol Biol Phys 2008, 72:S366.

18. Vanetti E, Clivio A, Nicolini G, Fogliata A, Ghosh-Laskar S, Agarwal JP, Upreti RR, Budrukkar A, Murthy V, Deshpande DD, et al: Volumetric modulated arc radiotherapy for carcinomas of the oro-pharynx, hypopharynx and larynx: A treatment planning comparison with fixed field IMRT. Radiother Oncol 2009, 92:111-117.

19. Popescu CC, Olivotto IA, Beckham WA, Ansbacher W, Zavgorodni S, Shaffer R, Wai ES, Otto K: Volumetric Modulated Arc Therapy Improves Dosimetry and Reduces Treatment Time Compared To Conventional Intensity-Modulated Radiotherapy for Locoregional Radiotherapy of LeftSided Breast Cancer and Internal Mammary Nodes. Int J Radiat Oncol Biol Phys 2010, 76:287-95.

20. Shaffer R, Nichol AM, Vollans E, Fong M, Nakano S, Moiseenko V, Schmuland M, Ma R, McKenzie M, Otto K: A Comparison of Volumetric Modulated Arc Therapy and Conventional Intensity-Modulated Radiotherapy for Frontal and Temporal High-Grade Gliomas. Int J Radiat Oncol Biol Phys 2009

21. Wagner D, Christiansen H, Wolff H, Vorwerk H: Radiotherapy of malignant gliomas: Comparison of volumetric single arc technique (RapidArc), dynamic intensity-modulated technique and 3D conformal technique. Radiother Oncol 2009, 93:593-596.

22. Wolff D, Stieler F, Welzel G, Lorenz F, Abo-Madyan Y, Mai S, Herskind C, Polednik M, Steil V, Wenz F, et al: Volumetric modulated arc therapy (VMAT) vs. serial tomotherapy, step-and-shoot IMRT and 3D-conformal RT for treatment of prostate cancer. Radiother Oncol 2009, 93:226-233.

23. Taylor A, Rockall AG, Reznek RH, Powell ME: Mapping pelvic lymph nodes: guidelines for delineation in intensity-modulated radiotherapy. Int J Radiat Oncol Biol Phys 2005, 63:1604-1612.

24. Gallagher MJ, Brereton HD, Rostock RA, Zero JM, Zekoski DA, Poyss LF, Richter MP, Kligerman MM: A prospective study of treatment techniques to minimize the volume of pelvic small bowel with reduction of acute and late effects associated with pelvic irradiation. Int J Radiat Oncol Biol Phys 1986, 12:1565-1573.

25. Mell LK, Kochanski JD, Roeske JC, Haslam JJ, Mehta N, Yamada SD, Hurteau JA, Collins YC, Lengyel E, Mundt AJ: Dosimetric predictors of acute hematologic toxicity in cervical cancer patients treated with concurrent cisplatin and intensity-modulated pelvic radiotherapy. Int J Radiat Oncol Biol Phys 2006, 66:1356-1365.

26. Mell LK, Schomas DA, Salama JK, Devisetty K, Aydogan B, Miller RC, Jani AB, Kindler $\mathrm{HL}$, Mundt AJ, Roeske JC, et al: Association between bone marrow dosimetric parameters and acute hematologic toxicity in anal cancer patients treated with concurrent chemotherapy and intensity-modulated radiotherapy. Int J Radiat Oncol Biol Phys 2008, 70:1431-1437.

27. Mell LK, Tiryaki H, Ahn KH, Mundt AJ, Roeske JC, Aydogan B: Dosimetric comparison of bone marrow-sparing intensity-modulated radiotherapy versus conventional techniques for treatment of cervical cancer. Int $J$ Radiat Oncol Biol Phys 2008, 71:1504-1510.

28. RTOG 0529: A phase II evaluation of dose-painted IMRT in combination with 5-fluorouracil and mitomycin- $C$ for reduction of acute morbidity in carcinoma of the anal canal. [http://rtog.org/members/protocols/0529/ 0529.pdf].

29. Emami B, Lyman J, Brown A, Coia L, Goitein M, Munzenrider JE, Shank B, Solin LJ, Wesson M: Tolerance of normal tissue to therapeutic irradiation. Int J Radiat Oncol Biol Phys 1991, 21:109-122.

30. Cozzi L, Dinshaw KA, Shrivastava SK, Mahantshetty U, Engineer R, Deshpande DD, Jamema SV, Vanetti E, Clivio A, Nicolini G, et al: A treatment planning study comparing volumetric arc modulation with
RapidArc and fixed field IMRT for cervix uteri radiotherapy. Radiother Oncol 2008, 89:180-191.

31. Shibamoto $Y$, Ito $M$, Sugie $C$, Ogino $H$, Hara M: Recovery from sublethal damage during intermittent exposures in cultured tumor cells: implications for dose modification in radiosurgery and IMRT. Int J Radiat Oncol Biol Phys 2004, 59:1484-1490.

32. Wang JZ, Li XA, D'Souza WD, Stewart RD: Impact of prolonged fraction delivery times on tumor control: a note of caution for intensitymodulated radiation therapy (IMRT). Int J Radiat Oncol Biol Phys 2003, 57:543-552.

33. Randall ME, Ibbott GS: Intensity-modulated radiation therapy for gynecologic cancers: pitfalls, hazards, and cautions to be considered. Semin Radiat Oncol 2006, 16:138-143.

34. Hall EJ: Intensity-modulated radiation therapy, protons, and the risk of second cancers. Int J Radiat Oncol Biol Phys 2006, 65:1-7.

35. Hall EJ, Wuu CS: Radiation-induced second cancers: the impact of 3D-CRT and IMRT. Int J Radiat Oncol Biol Phys 2003, 56:83-88.

36. Dorr W, Herrmann T: Second primary tumors after radiotherapy for malignancies. Treatment-related parameters. Strahlenther Onkol 2002, 178:357-362

doi:10.1186/1748-717X-5-92

Cite this article as: Vieillot et al:: Plan comparison of volumetricmodulated arc therapy (RapidArc) and conventional intensitymodulated radiation therapy (IMRT) in anal canal cancer. Radiation Oncology 2010 5:92.

\section{Submit your next manuscript to BioMed Central and take full advantage of:}

- Convenient online submission

- Thorough peer review

- No space constraints or color figure charges

- Immediate publication on acceptance

- Inclusion in PubMed, CAS, Scopus and Google Scholar

- Research which is freely available for redistribution

Submit your manuscript at www.biomedcentral.com/submit
C) Biomed Central 